\title{
An Architecture to Increase Performance of Self-Organizing Networks *
}

\author{
Fanilo Harivelo, Pascal Anelli \\ IREMIA, Université de La Réunion \\ BP 7151, 15 Avenue R. Cassin, 97715 Saint Denis Messag 9, France \\ \{Fanilo.Harivelo | Pascal.Anelli\}@univ-reunion.fr \\ Gwendal Le Grand \\ GET/Télécom Paris, LTCI-UMR 5141 CNRS, Département INFRES \\ 37-39 rue Dareau, 75014 Paris, France \\ gwendal.legrand@enst.fr
}

\begin{abstract}
The unpredictable variations of the characteristics of wireless and ad hoc networks make Quality of Service (QoS) support difficult. Wireless Mesh Networks constitute an alternative to provide QoS in an ad hoc network. This paper proposes a wireless mesh solution that reduces co-channel interference and simplifies both services and resources management. The architecture is based on a topology control or clustering scheme and a channel assignment mechanism. The network is organized hierarchically in order to obtain a seamless stable backbone. The clustering scheme aims to achieve a maximum number of covered nodes and a minimum hop count, while providing a topology minimizing co-channel interference by means of a channel assignment algorithm. Performance evaluation in NS-2 shows that resource management in a cluster can be uncoupled from that of neighboring clusters and that the network achieves improved performance compared to a classical ad hoc network.
\end{abstract}

\section{Introduction}

An ad hoc network is a self-organizing network of stations connected by wireless links. Quality of Service (QoS) management, which is a key problem in the current Internet, is made even more complicated in ad hoc environments due to the dynamicity of the topology and to the wireless characteristics of the links. One possible solution to support QoS in ad hoc network is to adopt a Wireless Mesh Network (WMN) structure [1]. A WMN is a dynamic, selforganized, and self-configured network in which nodes automatically form an ad hoc network and maintain mesh connectivity. It deals with the topology variation problem by offering a seamless stable backbone. Two types of nodes are defined in WMNs: mesh clients (or stations) and mesh routers (or routers). The latter ensure routing, have limited mobility and form a stable backbone for mesh clients. Clients support only lightweight functionalities while main functionalities are delegated to routers. The clustering process (or topology creation phase) is the organization of stations into clusters or cells [13] and placed under the authority of a router. Clustering consists in finding a suitable partitioning that satisfies specific objectives. The benefits of partitioning into cells is manifold: it allows spatial reuse of the frequency for non-overlapping cells, it provides a better coordination between stations (through the router) that eases routing (by organizing it hierarchically), and it offers a more stable backbone to stations that prevents variations in link quality.

Common clustering objectives focus on routing ([12], [3]), cluster maintenance ([8], [7]), energy ([2], [11]) and load balancing ([2]), but interference issues (which can degrade network performance in heavy load scenario), were never considered so far. However, we believe that an adequate channel assignment can reduce drastically interference between neighboring clusters, allow an individual resource management, and thus increase network performance. In order to reduce co-channel interference and facilitate resource control in wireless ad hoc networks, this paper proposes a wireless mesh architecture based both on a topology control model (or clustering scheme) and a channel assignment algorithm. Co-channel interferences are due to cells that operate within the same frequency. Graph coloring theory is generally used to solve the channel assignment problem. The main issue in graph coloring [10] is the $\mathrm{k}$-colorability of the graph, where $\mathrm{k}$ is the number of colors or channels. Determining the k-colorability of a graph is known to be NP-hard for $k \geq 3$. 
Contrary to classical channel assignment algorithms that apply to all types of graphs, our approach acts on a predefined set of graph classes that is constrained by the clustering scheme to be 3-colorable. Our clustering scheme is an extension of the model presented in [9]. and also introduces improved accuracy in the modelling. It offers a framework on which the execution of the proposed channel assignment algorithm will issue a 3-colorable network with minimum co-channel interference. In addition to the former model objectives, which are to achieve a maximum covered nodes number and a minimum hop count, the derived clustering scheme introduces interference constraints and proposes an improved modelling of the network. Our channel assignment algorithm performs step by step channel allocation to clusters defined by the clustering scheme. At each step, our scheme selects the most constrained cluster and assigns a channel to it.

The rest of the paper is organized as follows. Our solution is presented in section 2. The proposed model is evaluated in section 3. Finally, we conclude in section 4.

\section{Hierarchical organization of the network}

This paper introduces a hierarchical architecture, related to WMNs, in order to ease services and resources management. Our architecture is based on a stable high quality backbone formed by mobile routers having a long autonomy. It consists in arranging self-organized networks into sub-groups called clusters or cells [13] and placed under the authority of a node (mobile router).

The clustering scheme (the process by which cells are formed) aims to maximize the number of covered nodes and minimize the number of hops to improve delay experienced by communications. In the first level of the hierarchy, stations are gathered into cells. Bandwidth management within a cell must not interfere with that of neighboring cells. Given a number of routers, the whole coverage area must wrap a maximum of stations. Then, communications between cells are handled by mobile routers in the second level of the hierarchy. Naturally, this implies that the communication is not routed by neighbor stations but by the router that relays the communication out of its cell. Cell coverage is defined by the router's location; it represents the area within which a station can be served by the router which is assumed to be movable. A barebone can serve, for example, as a router. The deployment has to ensure a connected backbone, i.e., the network composed of routers must be connected, and hop counts must be minimized.

Station locations are assumed to be known. Three frequencies are available according to the standard [6]. A station can operate on one of the three possible frequencies and can shift to a new frequency when moving from a cell to an another. A router uses three interfaces.

The use of different frequencies in neighboring cells isolates intra-cell traffic and limits the impact of co-channel interference. This introduces an additional constraint: the graph obtained from cells determination must be colorable with three colors that correspond to the three frequencies. To tackle the communication between routers, the three interfaces in a router operate on the different frequencies used.

The proposition consists in two phases:

- The first phase aims to split the network into cells. This proposal determines routers deployment in order to offer a connected network with a maximum coverage, while ensuring that the resulting graph is 3-colorable. The additional 3-colorability condition aims to allow frequency reuse, increase network performance, minimize co-channel interference and separate the intracell control of resources.

- The second phase purpose is the graph coloring obtained from the first phase, thanks to a channel assignment algorithm. It defines the transmitting and the receiving frequency to be adopted in each cell.

\subsection{Clustering scheme}

The clustering scheme derives from the model presented in [9].

An ad hoc network is connected if and only if a path there exists at least one path between each pair of stations. In order to achieve connectivity, routers' locations have to be computed in such a way to maximize the number of covered stations and minimize the hop counts. A new constraint that corresponds to the 3-colorability of the graph formed by the set of cells, is introduced in our model. We state that a graph is colorable with 3 colors if no more than 3 cells are within the transmission range of each other.

The problem can be expressed by a Mixed Integer Linear Programming (MILP) model and a set of constraints.

\subsubsection{Graph modelling}

To model router deployment into a MILP problem, we use the following notations:

- N: Number of mobile stations in the network

- M: Number of mobile routers to be deployed

- $P_{i}\left(x_{i}, y_{i}\right):$ Coordinates of a station $M S_{i}, 1 \leq i \leq N$

- $R_{s}, R_{r}$ : Station and Router transmission range, respectively

- $\left[x_{\min }, x_{\max }\right] \mathrm{x}\left[y_{\min }, y_{\max }\right]:$ Deployment field 
- $Q_{i}\left(a_{i}, b_{i}\right):$ Coordinates of a router $M R_{i}, 1 \leq i \leq M$

In the following, the euclidean distance between two points, $I\left(x_{I}, y_{I}\right)$ and $J\left(x_{J}, y_{J}\right)$, is defined as: $d(I, J)=$ $\sqrt{\left(x_{J}-x_{I}\right)^{2}+\left(y_{J}-y_{I}\right)^{2}}$. Network connectivity is expressed via binary variables $\left(\lambda_{i j}, \tau_{i}, \mu_{i j}, z_{i j}^{s d}, \nu_{i j}\right)$. In order to be covered by a router $M R_{j}$, the distance between a station $M S_{i}$ and a router $M R_{j}$, must be less than $R_{s}$. The variable $\lambda_{i j}, 1 \leq i \leq N, 1 \leq j \leq M$ is then introduced:

$$
\lambda_{i j}=\left\{\begin{array}{lll}
1 & \text { if } & d\left(P_{i}, Q_{j}\right) \leq R_{s} \\
0 & \text { if } \quad d\left(P_{i}, Q_{j}\right)>R_{s}
\end{array}\right.
$$

To express the coverage state of a station, $M S_{i}$, the variable $\tau_{i}, 1 \leq i \leq N$ is defined:

$$
\tau_{i}= \begin{cases}1 & \text { if } \exists j, 1 \leq j \leq M, \quad \lambda_{i j}=1 \\ 0 & \text { otherwise }\end{cases}
$$

Thus, a station $M S_{i}$ is covered if it is covered by at least one router, otherwise it in uncovered, for $1 \leq i \leq N$. This can be expressed by:

$$
\lambda_{i j} \leq \tau_{i} \leq \sum_{k=1}^{M} \lambda_{i j}, \text { for } 1 \leq j \leq M
$$

Two routers $M R_{i}$ and $M R_{j}$ are adjacent or neighbors if the distance between is smaller than $R_{r}$. That leads to the definition of variable $\mu_{i j}, 1 \leq i, j \leq M$ :

$$
\mu_{i j}=\left\{\begin{array}{lll}
1 & \text { if } & d\left(Q_{i}, Q_{j}\right) \leq R_{r} \\
0 & \text { if } \quad & d\left(Q_{i}, Q_{j}\right)>R_{r}
\end{array}\right.
$$

This variable ensures the connectivity between two routers.

To check backbone connectivity, the existence of a route from any source $M R_{s}, 1 \leq s \leq M$ to any destination $M R_{d}, 1 \leq d \leq M$, is tested. This is done using variable $z_{i j}^{s d}, 1 \leq i, j, s, d \leq M$ :

$$
z_{i j}^{s d}=\left\{\begin{array}{c}
1 \quad \text { if } \exists \text { a route from } M R_{s} \text { to } M R_{d} \\
\text { via the link }\left(M R_{i}, M R_{j}\right) \\
0 \quad \text { otherwise }
\end{array}\right.
$$

with $z_{i i}^{s d}=0$ and $z_{i j}^{s s}=0$.

Thus, if two nodes are not connected, no link can be created between them. This is expressed by the following equation, $1 \leq i, j, s, d \leq M$ :

$$
z_{i j}^{s d} \leq \mu_{i j}
$$

The flow conservation principle, which states that the difference between incoming flows and outgoing flows for a node equals the node's demand, must apply to each router, $1 \leq i, s, d \leq M$ :

$$
\sum_{j=1}^{M} z_{i j}^{s d}-\sum_{j=1}^{M} z_{j i}^{s d}=\left\{\begin{array}{c}
0 \quad \text { if } \quad(i \neq s \text { and } i \neq d) \\
\text { or }((i=s \text { or } i=d) \text { and } s=d) \\
-1 \quad \text { if } i=s \text { and } s \neq d \\
1 \text { if } i=d \text { and } s \neq d
\end{array}\right.
$$

To ensure the 3-colorability of the graph, variable $\nu_{i j}$ $1 \leq i, j \leq M$ is defined:

$$
\nu_{i j}=\left\{\begin{array}{lll}
1 & \text { if } & d\left(Q_{i}, Q_{j}\right) \leq 3 R_{s} \\
0 & \text { if } \quad & d\left(Q_{i}, Q_{j}\right)>3 R_{s}
\end{array}\right.
$$

The graph is 3-colorable, if for each quadruplet $\left(M R_{i}, M R_{j}, M R_{k}, M R_{l}\right), 1 \leq i, j, k, l \leq M$, at most, three of the four corresponding cells are within the transmission range of each other. Two cells are neighbors in terms of transmission range if their centers are at a distance less than $3 R_{s}$ away. This is to ensure that their outer member stations do not interfere. For example, in figure 1, the graph is 3-colorable if the distance between router 3 and router 4 is greater than $3 R_{s}$. In this case, there are only 3 cells within the transmission range of each other.

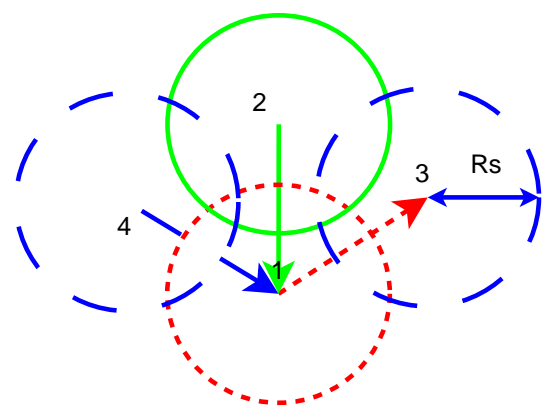

\section{Figure 1. 3-colorability and traffic manage- ment}

The four cells are within the transmission range of each other, if the $C_{4}^{2}=6$ couples are all neighbors. Inversely, they are not if, at least, one of the 6 couples is not neighbor. That brings us to the 3-colorability condition, for $1 \leq i, j, k, l \leq M$ and $\mathrm{i}, \mathrm{j}, \mathrm{k}, 1$ a disjoint set:

$$
\nu_{i j}+\nu_{i k}+\nu_{i l}+\nu_{j k}+\nu_{j l}+\nu_{k l}<6
$$

The objective that consists in a maximum number of covered stations and a minimum hop count leads to the maximization of the function:

$$
\sum_{i=1}^{N} \tau_{i}-\sum_{i, j, s} z_{i j}^{s 1}
$$

$\sum_{i=1}^{N} \tau_{i}$ counts the total number of covered stations (that has to be maximized). The substraction of $\sum_{i, j, s} z_{i j}^{s 1}$ forces routes to be as short as possible in the backbone formed by routers.

While the model must be expressed in MILP problem, constraints containing quadratic terms (equations (1), (3), (6)) have to be linearized. 


\subsubsection{Linearization of the quadratic constraints}

In the previous section, quadratic constraints (Equations (1), (3) and (6)) comprising euclidean distance, are in the form:

$$
\gamma=\left\{\begin{array} { l l l } 
{ 1 } & { \text { if } } & { d ( I , J ) \leq R } \\
{ 0 } & { \text { if } \quad d ( I , J ) > R }
\end{array} \Leftrightarrow \left\{\begin{array}{l}
\gamma=1 \Rightarrow d(I, J) \leq R \\
\gamma=0 \Rightarrow d(I, J)>R
\end{array}\right.\right.
$$

with $\gamma$ equals $\lambda_{i j}$ (resp. $\mu_{i j}$ or $\nu_{i j}$ ) and R corresponds to $R_{s}$ (resp. $R_{r}, 3 R_{s}$ ). We propose to linearize the quadratic constraints by using an octagon instead of a circular geometric representation. The center of the circle is moved to point I, and the couple $(\mathrm{X}, \mathrm{Y})$ represents the coordinates of point $\mathrm{J}$ in this new reference. The octagon will be formed by lines which are represented in figure 2 .

Eight inequalities verify the first condition of (9) and eight others verify the second condition. To tackle contradictions among these eight last inequalities (no intersection area for $\gamma=0$ ), we introduce new binary variables $\beta_{k}$, $1 \leq k \leq 8$ and an additional constraint. Then, we obtain the following system:

$$
\left\{\begin{array}{l}
\text { (L1): }-X \leq r+(1-\gamma) x_{\max } \\
\text { (L2): } X \leq r+(1-\gamma) x_{\max } \\
\text { (L3): }-Y \leq r+(1-\gamma) y_{\max } \\
\text { (L4): } Y \leq r+(1-\gamma) y_{\max } \\
\text { (L5): } Y+X \leq D+(1-\gamma) p_{\max } \\
\text { (L6): }-(Y+X) \leq D+(1-\gamma) p_{\max } \\
\text { (L7): } Y-X \leq D+(1-\gamma) p_{\max } \\
\text { (L8): }-(Y-X) \leq D+(1-\gamma) p_{\max } \\
\text { (L1): }-X \geq r-\beta_{1} x_{\max } \\
\text { (L2): } X \geq r-\beta_{2} x_{\max } \\
\text { (L3): }-Y \geq r-\beta_{3} y_{\max } \\
\text { (L4): } Y \geq r-\beta_{4} y_{\max } \\
\text { (L5): } Y+X \geq D-\beta_{5} p_{\max } \\
\text { (L6): }-(Y+X) \geq D-\beta_{6} p_{\max } \\
\text { (L7): } Y-X \geq D-\beta_{7} p_{\max } \\
\text { (L8): }-(Y-X) \geq D-\beta_{8} p_{\max } \\
\sum_{k=1}^{8} \beta_{k}-7 \leq \gamma \leq \frac{\sum_{k=1}^{8} \beta_{k}}{8}
\end{array}\right.
$$

where $p_{\max }=x_{\max }+y_{\max }, r=R \cos \frac{\pi}{8}, D=R\left(\sin \frac{\pi}{8}+\right.$ $\left.\cos \frac{\pi}{8}\right)$

\subsection{Frequency management}

In the above controlled network, communications in a cell are isolated from those of neighboring cells by using different channels. Thus, two adjacent cells must not use the same channel. The basic idea of the frequency assignment is to color from the crowded region to the edge. This is done by coloring the already colored region and prioritizing by order, constrained vertices, partially colored regions and then crowded uncolored regions. A neighbor of a vertex in the algorithm is a vertex that must have a different color

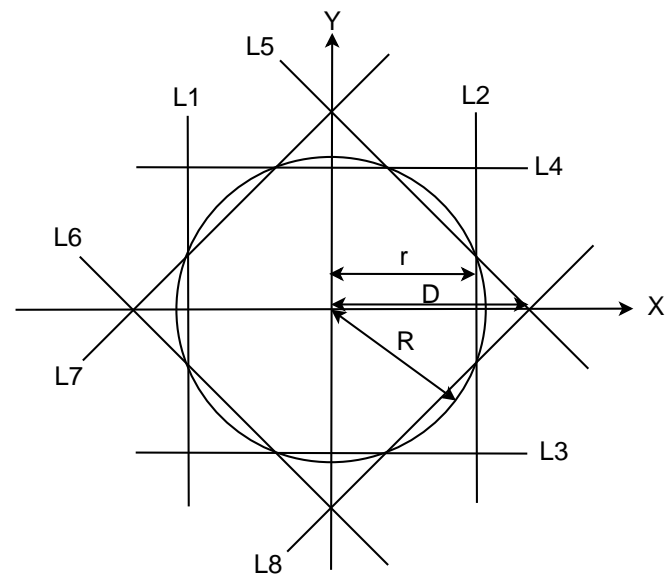

Figure 2. Linearization of a euclidean distance

(the same as the one defined in the previous section). The vertices' coloring ordering is determined according to the following comparison criteria, sorting by priority and corresponding each to a parameter: Coloring state (colored or uncolored), Colored neighbors number in a clique, Clique size, Colored neighbors number and Neighbors number

At each step, the following algorithm is used:

- Initially, all vertices are uncolored.

- 1. Pick and color the first vertex of the remaining uncolored vertices list at each step using greedy fashion with color ordering $(1,2,3)$, i.e., pick the first color in this order that satisfies channel constraints. It consists in taking the first color of the list that satisfies frequency constraints.

- 2. Update neighbors' parameters.

- 3. If all vertices are colored, exit.

- 4. Resort the remaining uncolored vertices

\section{- 5. Goto 1 .}

The transmitting channel of a station is the same as that of the router serving its cell. Inter-cell transmissions are handled by routers. At most, a router can receive on two interfaces while transmitting on the third or receive simultaneously on the three interfaces. For instance, in figure 1, router 1 can transmit to router 3 on router 1's channel and receive transmissions from routers 2 and 4 . 


\section{Evaluation}

Evaluation is conducted on a IEEE 802.11 wireless network composed of 50 stations. We consider a 1000x 1000 $\mathrm{m}^{2}$ field. Five routers are proposed to cover a maximum number of stations. Stations and routers ranges equal respectively, $250 \mathrm{~m}$ and $500 \mathrm{~m}$.

\subsection{Clustering and frequency assignment}

CPLEX [5] is used to solve linear programming problems which are expressed using the AMPL [4] language. The channel assignment algorithm is applied to the resulting deployment. Then, we obtain the network partitioning represented in figure 3.

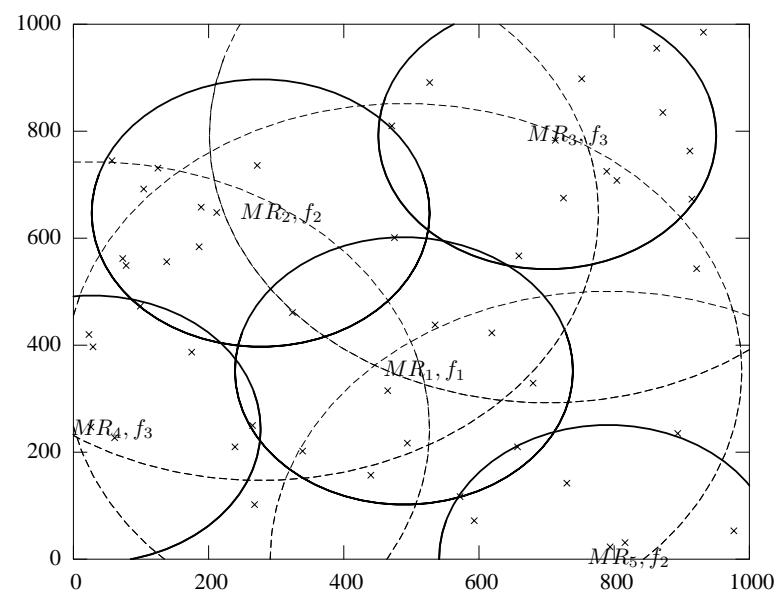

Figure 3. Resulting topology of clustering and channel assignment algorithm

Circles in plain lines represent cell coverages while router transmission ranges are represented by dashed circles. $\left(M R_{i}, f_{j}\right)$ relates to router index $i$ and the channel $j$ used within the cluster served by this router. One notices that only 4 stations remain uncovered. A possible solution is to introduce an additional router. Another solution is to let uncovered stations access the network through the other covered stations.

\subsection{Performance evaluation}

To evaluate performance of the proposed model, simulations are performed on the resulting network under NS-2. The network capacity equals $1 \mathrm{Mbits} / \mathrm{s}$. Five other stations are added to the topology at the locations of the routers, in order obtain similar network conditions. These stations do not generate any traffic. For each simulation, we consider two cases: the first one corresponds to a classical ad hoc network and the second one to the proposed architecture applied to the same topology. In the second case, the five additional stations are upgraded to routers.

\subsubsection{Intra-cluster communication}

The purpose of this simulation is to show the independence between intra-cell traffics. Three sources generate CBR traffic (flow 1, flow 2, and flow 3) at a rate of $700 \mathrm{kbits} / \mathrm{s}$, $600 \mathrm{kbits} / \mathrm{s}$ and $500 \mathrm{kbits} / \mathrm{s}$ respectively. Both flow sender and receiver are located in cell covered by $M R_{4}, M R_{2}$ and $M R_{3}$, respectively.

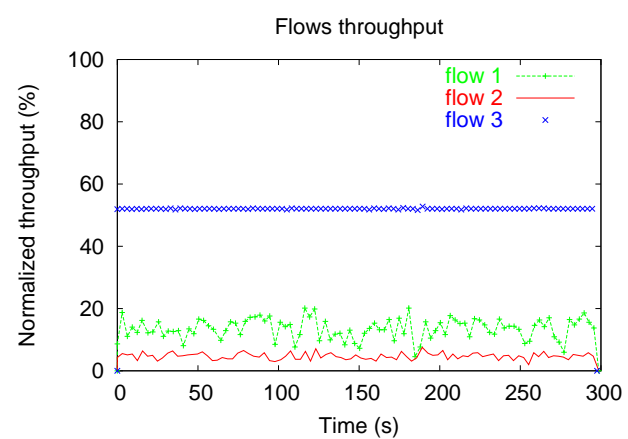

Figure 4. Flows throughput in the classical ad hoc network

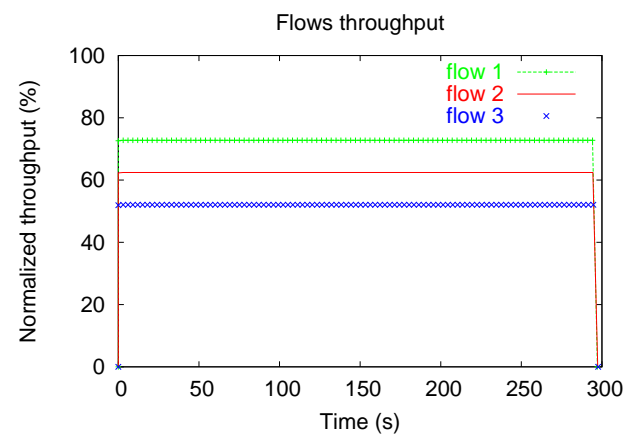

Figure 5. Flows throughput in the ad hoc network with the proposition

In the classical case (Fig. 4), the flow 1 (resp. flow 2) sender is a hidden station for the flow 2 (resp. flow 3 ) sender. This explains the throughput degradation of both flow 1 and flow 2 (Fig. 4). With the channel assignment (Fig. 5), the proposed architecture achieves independence between the three flows. Separate resource management is then possible within each cluster. 


\subsection{Intra-cluster and inter-cluster com- munication}

This evaluation aims to measure the performance of the architecture on arbitrary traffic pattern. A set of 60 simulations is performed. For each simulation, 15 couples of communicating stations are randomly chosen. Each sender generates a $500 \mathrm{kbits} / \mathrm{s}$ CBR traffic with a 512 bytes packet size . A simulation lasts $1000 \mathrm{~s}$.

Table 1 show encountered improvements in terms of the increase (resp. the decrease) of the throughput percentage (resp. delay or variation around the mean) with a margin of error equals $5 \%$.

\begin{tabular}{|c|c|c|}
\hline Metric & Mean value (\%) & Variation (\%) \\
\hline Throughput & $78.5_{-}^{+} 8.7$ & $-71.5_{-}^{+} 3.7$ \\
Delay & $-98.3_{-}^{+} 0.3$ & $-99.5_{-}^{+} 0.2$ \\
\hline
\end{tabular}

\section{Table 1. Flows mean throughput and mean delay with their respective standard deviation with a confidence interval of $95 \%$}

Improvements consist in an increase of a mean value of about $80 \%$ for the throughput and a reduction of about $90 \%$ for the delay. More stability is also observed for these two metrics: flows experience an improvement expressed by an average decrease of the variation around the mean, an increase by more than a factor 3 for throughput and by a factor 100 for delay. The delay decrease is mainly due to the little number of hops while the increased throughput comes from a mitigation of co-channel interference. Thus, the bandwidth is efficiently used.

\section{Conclusion}

This paper presented a wireless mesh architecture based on a topology control scheme or clustering scheme and a channel assignment mechanism. The purpose of the proposition consists in offering a seamless stable backbone for wireless stations. To improve bandwidth efficiency, a channel assignment algorithm is introduced in such a way to reduce co-channel interference. A first set of simulations shows that intra-cell communications are independent. For the second set of 60 simulations with randomly chosen traffic matrices, the proposed model achieves a betterment of about $80 \%$ for the throughput and $90 \%$ for the delay compared to a classical ad hoc network. Improvement of the throughput and delay stability is also observed (decrease of a factor 3 and 100, respectively). These performance improvements are due to reduced dependence between intracell communications and the smaller number of hops. The proposed architecture relates to cellular structure and is scalable to cover a large area. A router can be seen as an access point. An ad hoc network can then be settled while having an infrastructure mode at the stations' level. Thus, resource control can be decoupled from intra-cell and intercell levels. Our future works will focus on controlling bandwidth at each level of the proposed hierarchy.

\section{References}

[1] I. F. Akyildiz and X. Wang. A Survey on Wireless Mesh Networks. IEEE Communications Magazine, September 2005.

[2] A. D. Amis and R. Prakash. Load-Balancing Clusters in Wireless Ad Hoc Networks. Proceedings of the 3rd IEEE Symposium on Application-Specific Systems and Software Engineering Technology (ASSET'00), page 25, 2000.

[3] Y. P. Chen and A. L. Liestman. Approximating minimum size weakly-connected dominating sets for clustering mobile ad hoc networks. Proceedings of the 3rd ACM international symposium on Mobile ad hoc networking \& computing, Lausanne, Switzerland, pages 165-172, 2002.

[4] http://www.ampl.com. A Mathematical Programming Language.

[5] http://www.ilog.com/products/cplex. ILOG CPLEX 9.0 User's manual, October 2003.

[6] IEEE 802.11 WG. Wireless LAN medium access control (MAC) and physical layer (PHY) specifications. IEEE standard 802.11, 1999 Edition, 1999.

[7] T. J. Kwon and M. Gerla. Efficient Flooding with Passive Clustering (PC) in Ad Hoc Networks. ACM SIGCOMM Computer Communication Review, 32(1):44-56, January 2002.

[8] C. R. Lin and M. Gerla. Adaptive Clustering for Mobile Wireless Networks. IEEE Journal of Selected Areas in Communications, 1999.

[9] R. Meraihi, G. L. Grand, N. Puech, M. Riguidel, and $\mathrm{S}$. Tohm. Improving ad hoc network performance with backbone topology control. EEE Vehicular Technology Conference (VTC Fall 2004), Los Angeles, USA, September 26-29 2004.

[10] L. Narayanan. Channel assignment and graph multicoloring, chapter 1, pages $71-94$. John Wiley \& Sons, Inc. New York, NY, USA, 2002.

[11] J. Wu, M. Gao, and I. Stojmenovic. On Calculating PowerAware Connected Dominating Sets for Efficient Routing in Ad Hoc Wireless Networks. Proceedings of the 2001 International Conference on Parallel Processing, pages 346-356, 2001.

[12] J. Wu and H. Li. On calculating connected dominating set for efficient routing in ad hoc wireless networks. Proceedings of the 3rd international workshop on Discrete algorithms and methods for mobile computing and communications, Seattle, Washington, United States, pages 7-14, 1999.

[13] J. Y. Yu and P. H. J. Chong. A Survey of Clustering Schemes for Mobile Ad Hoc Networks. IEEE Communications Surveys and Tutorials, 7(1):32-48, First Quarter 2005. 